\title{
Postpartum Infection in Morbidly Obese Women after Caesarean Section: Does Early Prophylactic Oral Antibiotic Use Make a Difference?
}

\author{
Hannah Yeeles ${ }^{1}$, Sarah Trinick ${ }^{1}$, Charmaine Childs ${ }^{2}$, Hora Soltani' ${ }^{2}$, Tom Farrell ${ }^{3}$ \\ ${ }^{1}$ Rotherham General Hospital, NHS Foundation Trust, Rotherham, UK \\ ${ }^{2}$ Centre for Health and Social Care Research, Sheffield Hallam University, Sheffield, UK \\ ${ }^{3}$ Jessop Wing, Sheffield Teaching Hospitals NHS Trust, Sheffield, UK \\ Email: ${ }^{*}$.y.yeeles@doctors.org.uk
}

Received 13 May 2014; revised 10 June 2014; accepted 16 June 2014

Copyright (C) 2014 by authors and Scientific Research Publishing Inc.

This work is licensed under the Creative Commons Attribution International License (CC BY). http://creativecommons.org/licenses/by/4.0/

c) (i) Open Access

\section{Abstract}

The rising prevalence of morbid obesity particularly in women coupled with a higher likelihood of having a caesarean section (C-section) birth and an increased risk of surgical site infection (SSI) places wound management among priority areas in maternity care. There is ambiguity about the efficacy of routine preventative care pathways particularly in morbid obese women with regards to SSI after caesarean section. A pilot study was therefore undertaken to explore the number of women with a C-section infection in a cohort of morbidly obese women during six weeks postpartum against a protocol of standard care of early antibiotic prophylaxis and skin closure practice. A short questionnaire was sent to 59 women with an early pregnancy BMI $\geq 40$ who gave birth via C-section in a large maternity unit in Sheffield, UK. Data were collated from 39 women with 20 $(51 \%)$ developing a post-operative wound infection within 6 weeks postpartum. Infections were higher in the women who had emergency $C$-section births $(14 / 24,60 \%)$. There was no significant difference in wound infection risk with respect to wound closure material (Chi-square $=0.298$, p-value $=0.86$ ) or the use of oral prophylactic antibiotic after birth (Chi-square $=0.2053$, $p$-value $=$ 0.650). Although all the women received routine intravenous (IV) antibiotics before C-section, only 26/39 received the 5-day oral antibiotic prophylaxis after birth. Six of 13 women who did not receive postpartum oral antibiotics $(46 \%)$ developed a SSI. In summary, over half of morbidly obese women who delivered by C-section developed a wound infection, despite receiving prophylactic antibiotics. We acknowledge the limitations of these results from a small-sample retrospective observational study. However, this may indicate that post operative antibiotic prophylaxis confers

\footnotetext{
"Corresponding author.
} 
no additional benefit in this group of patients and thus requires further investigation.

Keywords

Wound, Surgical Site Infection, Morbidly Obese, Caesarean Section

\section{Introduction}

The rising prevalence of morbid obesity particularly in women [1] coupled with a higher likelihood of having a caesarean section (C-section) birth [2] and an increased risk of surgical site infection (SSI) [3] places wound assessment among priority areas in maternity care. Caesarean birth is the single most important risk factor for puerperal infection with BMI being a significant independent risk factor for SSI [4]. Local maternal care guidelines include the use of prophylactic antibiotics at induction of anaesthesia (IV) for all women, and for 5 days after delivery (oral) in those with a BMI $\geq 40\left(\mathrm{~kg} / \mathrm{m}^{2}\right)$. However, the efficacy is uncertain [5] since many women may develop infection after discharge from hospital with no routine tracking systems in place [4]. A pilot study was therefore undertaken to explore the number of women with a C-section infection in a cohort of morbidly obese women during six weeks postpartum against a background of standard care of early antibiotic prophylaxis and skin closure practice.

\section{Methods}

As part of a service evaluation, a short questionnaire was sent to 59 women with an early pregnancy BMI $\geq 40$ who gave birth via C-section in Jessop Wing Maternity Hospital, Sheffield, UK from November 2011 to October 2012. Approval was granted by the local clinical effectiveness unit.

\section{Results}

Of 59 questionnaires, data is available from 39 women aged 31.4 (5.9) years and with a BMI of 44.4 (6.5) (mean (SD)). Fifteen (38\%) women were delivered by elective C-section (indications: one or more previous C-sections, breech presentation, previous traumatic delivery) and 24 (62\%) by emergency C-section (indications: failure to progress in labour, fetal distress, failed induction of labor, pre-eclampsia).

Of 39 patients, 20 (51\%) developed a post-operative wound infection within 6 weeks postpartum. Infections were higher in the women who had emergency C-section births (14/24, 60\%). Half of women with a previous C-section (6/12) had a wound infection. Three (60\%) of the five women with gestational diabetes developed an infection. There was no significant difference in wound infection risk with respect to wound closure material (Chi-square $=0.298$, p-value $=0.86$ ) or the use of oral prophylactic antibiotic after birth (Chi-square $=0.2053$, p-value $=0.650)($ Table 1$)$.

Although all the women received routine IV antibiotics before C-section, only 26/39 received the 5 day oral antibiotic prophylaxis after birth. Six of 13 women who did not receive postpartum oral antibiotics (46\%) developed a SSI (Table 1).

\section{Discussion and Conclusions}

In summary, over half of morbidly obese women who delivered by C-section developed a wound infection, despite receiving prophylactic antibiotics.

Whilst recognising the limitations of these results from a small sample size, if this finding does exist for the larger population of morbidly obese women undergoing C-section, there is a suggestion that routine prophylactic antibiotic use does not confer significant benefit in reducing infection rates. That said, we accept the further limitations of our observations with respect to a lack of a control group (e.g. BMI 30 - 40) and hence the interpretation of the results outside of the specified group of morbidly obese women. Nevertheless, the observations take a first step in alerting the medical community, already concerned about antibiotic resistance, about SSI risk in this group of postpartum women. 
Table 1. Surgical site infection in morbidly obese women with respect to skin closure materials and the use of prophylactic antibiotics.

\begin{tabular}{ccc}
\hline & Number of women with infection & No infection reported \\
\hline Monocryl $^{\mathrm{TM}}$ & Skin closure material & 13 \\
Beaded Prolene $^{\mathrm{TM}}$ & 14 & 4 \\
Staples & 4 & 2 \\
& 2 & 12 \\
Antibiotic prophylaxis & Use of oral prophylactic antibiotics & 7 \\
No antibiotic prophylaxis & 14 & \\
\hline
\end{tabular}

Currently there is no consensus on wound surveillance and tracking within the maternal care pathway to identify and manage wound infection after discharge from hospital other than by self-report. More work is needed to examine the risk, the treatment pathways and outcome of current wound management and outcomes of prophylaxis, especially after discharge from hospital in this high-risk group of new mothers.

\section{References}

[1] Health and Social Care Information Centre (2013) The Health Survey for England—2012 Trend Tables. Health and Social Care Information Centre, London. http://www.hscic.gov.uk/catalogue/PUB13219

[2] Mantakas, A. and Farrell, T. (2010) The Influence of Increasing BMI in Nulliparous Women on Pregnancy Outcome. European Journal of Obstetrics Gynecology and Reproductive Biology, 153, 43-46. http://dx.doi.org/10.1016/j.ejogrb.2010.06.021

[3] National Institute of Clinical Excellence (NICE) (2008) Surgical Site Infection: Prevention and Treatment. National Institute of Clinical Excellence, London. http://www.nice.org.uk/nicemedia/live/11743/42378/42378.pdf

[4] Wloch, C., Wilson, J., Lamagni, T., Harrington, P., Charlett, A. and Sheridan, E. (2012) Risk Factors for Surgical Site Infection Following Caesarean Section in England: Results from a Multicentre Cohort Study. BJOG, 119, 1324-1333. http://dx.doi.org/10.1111/j.1471-0528.2012.03452.x

[5] Smaill, F.M. and Gyte, G.M. (2010) Antibiotic Prophylaxis versus No Prophylaxis for Preventing Infection after Cesarean Section. Cochrane Database of Systematic Reviews, 1, CD007482. 